\title{
Probing the low-frequency vortex dynamics in a nanostructured superconducting strip
}

\author{
C. C. de Souza Silva, ${ }^{1}$ B. Raes, ${ }^{2}$ J. Brisbois,${ }^{3}$ L. R. E. Cabral, ${ }^{1}$ A. V. Silhanek, ${ }^{3}$ J. Van de Vondel, ${ }^{2}$ and V. V. Moshchalkov ${ }^{2}$ \\ ${ }^{1}$ Departamento de Física, Universidade Federal de Pernambuco, Cidade Universitária, 50670-901 Recife-PE, Brazil \\ ${ }^{2}$ INPAC - Institute for Nanoscale Physics and Chemistry, Department of Physics and Astronomy, KU Leuven, \\ Celestijnenlaan 200D, B-3001 Leuven, Belgium \\ ${ }^{3}$ Département de Physique, Université de Liège, Allée du 6 août 19, B5a, B-4000 Sart Tilman, Belgium
}

(Received 19 January 2016; revised manuscript received 11 March 2016; published 20 July 2016)

\begin{abstract}
We investigate by scanning susceptibility microscopy the response of a thin $\mathrm{Pb}$ strip, with a square array of submicron antidots, to a low-frequency ac magnetic field applied perpendicularly to the film plane. By mapping the local permeability of the sample within the field range where vortices trapped by the antidots and interstitial vortices coexist, we observed two distinct dynamical regimes occurring at different temperatures. At a temperature just below the superconducting transition, $T / T_{c}=0.96$, the sample response is essentially dominated by the motion of highly mobile interstitial vortices. However, at a slightly lower temperature, $T / T_{c}=0.93$, the interstitial vortices freeze up leading to a strong reduction of the ac screening length. We propose a simple model for the vortex response in this system which fits well to the experimental data. Our analysis suggests that the observed switching to the high mobility regime stems from a resonant effect, where the period of the ac excitation is just large enough to allow interstitial vortices to thermally hop through the weak pinning landscape produced by random material defects. This argument is further supported by the observation of a pronounced enhancement of the out-of-phase response at the crossover between both dynamical regimes.
\end{abstract}

DOI: 10.1103/PhysRevB.94.024516

\section{INTRODUCTION}

The dynamics of vortices in type-II superconductors strongly depends on the nature and distribution of disorder [1]. A powerful method to probe the physical mechanisms ruling the dynamics and dissipation of these systems consists in disturbing the vortex ensemble periodically (e.g., by an oscillating magnetic field or current) at a time scale comparable with the characteristic time of the dynamical process under consideration while simultaneously acquiring the in-phase and out-of-phase responses [2]. Since the vast majority of the techniques implementing the above described method involve large amounts of vortices, theoretical models need to be applied to derive, in most cases, a rather crude picture of the microscopic vortex physics (see, for instance, Ref. [3] and references therein).

The ac-susceptibility technique uses a small alternating magnetic field excitation and measures the total phase-locked flux change through a pickup coil surrounding the whole sample [4]. Traditionally one records the in-phase and outof-phase magnetic response, as they are related respectively to the macroscopic shielding abilities and the overall energy losses in the sample [5]. The drawback of using such a global method is the indirect relationship with the periodic motion of the vortices within the sample, as this zero-mean displacement does not result in a net flux change. However, the pickup coil is sensitive to possible changes in the ac-penetration depth (i.e., the total sample's impedance or screening ability) induced by the vortex dynamics. As such, macroscopic ac-susceptibility measurements can provide us only indirect, sample-averaged information about the microscopic vortex motion.

The incapacity to resolve the ac response of a single vortex and the indirect relation between the vortex dynamics and the integrated response can be circumvented by the scanning ac-susceptibility microscopy (SSM) technique [6-8], which allows one to probe the in- and out-of-phase response to an applied ac magnetic field or current with singlevortex resolution $[3,8,9]$. In contrast to standard susceptibility measurements, in SSM the periodic change in flux due to vortex motion and screening currents is measured locally by scanning a submicron-sized Hall probe over the surface, thereby revealing the microscopic vortex response. Moreover, the screening current response imaged close to the border, also probed in classical macroscopic ac-susceptibility experiments, can be cross-correlated with the present vortex physics and the theoretical models describing them.

In this work, we use the SSM technique to investigate the response of a nanostructured superconductor having a square lattice of antidots. A prominent feature of these systems is the typically very different mobility of vortices strongly pinned at the antidots and those sitting at the interstitial positions, which probe a smooth "cage" potential produced by the vortices at the antidots [10-12]. This reflects in different (according to whether or not interstitial vortices are present) scenarios of the sample response to an excitation, as indeed observed in a great deal of macroscopic ac-susceptibility experiments [13-22]. The interpretation of the different dynamical regimes usually ignores the random pinning potential produced by sample inhomogeneities, considered negligible at the temperatures where the experiments are carried out. However, at least for experiments performed within the linear regime, where vortices are driven close to their equilibrium positions by very small driving forces, the weak material pinning might become important or even dominant.

Although the effect of quenched disorder in hard-pinning, nanostructured $\mathrm{Nb}$ films has already been investigated [23], it should be noted that, so far, the interplay of all relevant energy scales, vortex-antidot, vortex caging, vortex-random pinning, and thermal fluctuations has not been fully addressed, neither experimentally nor theoretically. Here, we take profit of the local character of the SSM technique to tackle this problem on scales smaller than the screening length, which allows one 
to map in detail the contribution of the vortex dynamics to the ac permeability for different fields and temperatures. We consider a nanostructured film made of $\mathrm{Pb}$, a well-known softpinning material, and find that pinning by material defects in this sample, as well as thermal activation processes, dominate the low-frequency linear response even at temperatures very close to $T_{c}$, where quenched disorder is traditionally neglected.

The paper is organized as follows. In Sec. II, we revisit the general problem of the linear response of a superconducting strip and propose a simple method to calculate the ac magnetic permeability in terms of the complex ac screening length $\left(\Lambda_{a c}\right)$, which carries all essential information about the acdriven vortex motion. Then, we extend the two-species model introduced in Ref. [24] to derive a formula for $\Lambda_{a c}$ in terms of the vortex-antidot, vortex-cage, and vortex-defects coupling constants. This sets the general theoretical framework within which the experimental results will be interpreted. The results of the SHPM experiments, performed in both dc and ac modes, are presented in Sec. III. In Sec. IV we discuss the procedure for fitting the model to the experimental data, which allows us to quantitatively estimate all relevant elastic constants and thereby unveil the interplay between all these energy scales. The conclusions are presented in Sec. V.

\section{THEORETICAL FRAMEWORK}

\section{A. Linear response of a thin strip}

We consider a thin superconducting strip of width $2 a$ and thickness $d \ll \lambda$, subjected initially to a perpendicular dc magnetic field $\mathbf{H}=H_{d c} \hat{z}$ and define the superconducting sheet current $\mathbf{J}=\int d z \mathbf{j} \simeq \mathbf{j} d$, with $\mathbf{j}$ the local supercurrent density. In such geometry, the stationary sheet current distribution satisfies the nonlocal London equation [25]

$$
\Lambda \frac{\partial J}{\partial x}=H_{d c}-\mu_{0}^{-1} B_{v}+\frac{1}{2 \pi} \int_{-a}^{a} \frac{J\left(x^{\prime}\right)}{x-x^{\prime}} d x^{\prime},
$$

where $\Lambda=\lambda^{2} / d$ is the effective London penetration depth; $B_{v}$ is the local flux density induced by vortices. The nonlocal character of Eq. (1) comes from the last term, which corresponds to Ampère's law and gives the magnetic field produced by the sheet current flowing in the superconductor. However, we are interested in finding the (steady state) time evolution of the sheet current as the system is dynamically disturbed by an alternating drive. That is, we seek for an ac version of Eq. (1). For this, we follow below a procedure similar to that described in Ref. [26].

One can establish the connection between the vortex dynamics and the electromagnetic fields in a superconductor with the London equation relating the electric field and the current density [27]

$$
\mathbf{E}=\mathbf{B}_{v} \times \mathbf{v}+\mu_{0} \lambda^{2} \frac{\partial \mathbf{j}}{\partial t},
$$

where the first term represents the electric field induced by the moving vortices (with local mean speed $\mathbf{v}$ and flux density $\mathbf{B}_{v}$ ) averaged over scales larger than the intervortex spacing, while the second term accounts for the contribution of the Meissner effect. This local relation is due to the fact that in the first term the main contribution for the electric field comes from processes occurring inside the vortex cores [28].
When a sufficiently small excitation $\mathbf{h}(t)=\hat{z} h_{a c} e^{i \omega t}$ is superimposed with the dc field, the induced sheet current takes the form $\mathbf{J}(t)=\mathbf{J}_{d c}+\mathbf{J}_{a c}(\omega) e^{i \omega t}$, where $\mathbf{J}_{a c}(\omega)$ is in general complex, which accounts for a possible phase lag with respect to $\mathbf{h}(t)$. In addition, within the mean-field approximation, vortices respond linearly and the time dependence of their displacement field can be expressed quite generally as $\mathbf{u}(t)=$ $\mathbf{u}(\omega) e^{i \omega t}$, with [2,29,30]

$$
\mathbf{u}(\omega)=\mu_{v}(\omega) \mathbf{J}_{a c}(\omega) \times \hat{z},
$$

where $\mu_{v}(\omega)$ is the vortex response function. In this limit, $\mathbf{v}(t)=i \omega \mu_{v}(\omega) \mathbf{J}_{a c} \times \hat{z} e^{i \omega t}$ and $\partial \mathbf{j} / \partial t \simeq i \omega\left(\mathbf{J}_{a c} / d\right) e^{i \omega t}$, which by substitution in Eq. (2) leads to a complex Ohm's law (here expressed in the frequency domain):

$$
\mathbf{E}(\omega)=i \omega \mu_{0} \Lambda_{a c}(\omega) \mathbf{J}(\omega),
$$

where $\Lambda_{a c}(\omega)$ is the complex screening length, given by

$$
\Lambda_{a c}(\omega)=\Lambda+\Lambda_{v}(\omega), \quad \text { with } \quad \Lambda_{v}(\omega)=\frac{B_{v}}{\mu_{0}} \mu_{v}(\omega) .
$$

At this point, we will further assume a uniform vortex distribution (uniform $B_{v}$ ), as is approximately the case for field-cooled experiments. Within this approximation, $\Lambda_{a c}$ is a position independent parameter. In fact, as we shall discuss later (cf. Sec. III B), the vortex distribution can be depleted within a distance $\sim \Lambda$ close to the sample edge. This leads to a position-dependent $\Lambda_{v}$. However, in our sample, the effect of this flux depleted region can be neglected (see the Supplemental Material [31] for more details).

Within the approximations described above, the ac vortex dynamics can be inferred from measurements of the effective impedance (ac resistivity) of the sample, since $\rho_{a c}(\omega)=$ $i \omega \mu_{0} d \Lambda_{a c}(\omega)$. For instance, for the cases where vortices are immobile due to very strong pinning or absent in the sample, $\rho_{a c}=i \omega \mu_{0} \lambda^{2}$ is purely imaginary and the sample is in a dissipation-free Meissner state. More generally, however, vortex motion induces dissipation resulting in an additional, complex screening length, $\Lambda_{v}(\omega)$, which ultimately leads to a phase-lagged (complex) current distribution throughout the sample.

For a long, thin strip, $\mathbf{J}=J(x) \hat{y}$ by symmetry and the relation between the $z$ component of the local magnetic induction, $b_{z}(x)$, and the sheet current, $J(x)$, follows from Ampère's law:

$$
\mu_{0}^{-1} b_{z}(x)=H+\frac{1}{2 \pi} \int_{-a}^{a} \frac{J\left(x^{\prime}\right)}{x-x^{\prime}} d x^{\prime} .
$$

By taking the time derivative of Eq. (6) and using Faraday's law, $\dot{b}_{z}=-\partial E / \partial x$, and the material relation [Eq. (4)], one obtains an equation for $J_{a c}(x, \omega)$ :

$$
\Lambda_{a c} \frac{\partial J_{a c}}{\partial x}=h_{a c}+\frac{1}{2 \pi} \int_{-a}^{a} \frac{J_{a c}\left(x^{\prime}, \omega\right)}{x-x^{\prime}} d x^{\prime} .
$$

This equation is formally identical to the Meissner $\left(B_{v}=0\right)$ version of Eq. (1), except that here the effective penetration depth, $\Lambda$, is replaced by the complex screening length, $\Lambda_{a c}$, and the external dc field is replaced by the amplitude of the ac excitation, $h_{a c}$. The integral form of Eq. (7) was derived before by Brandt [26]. 


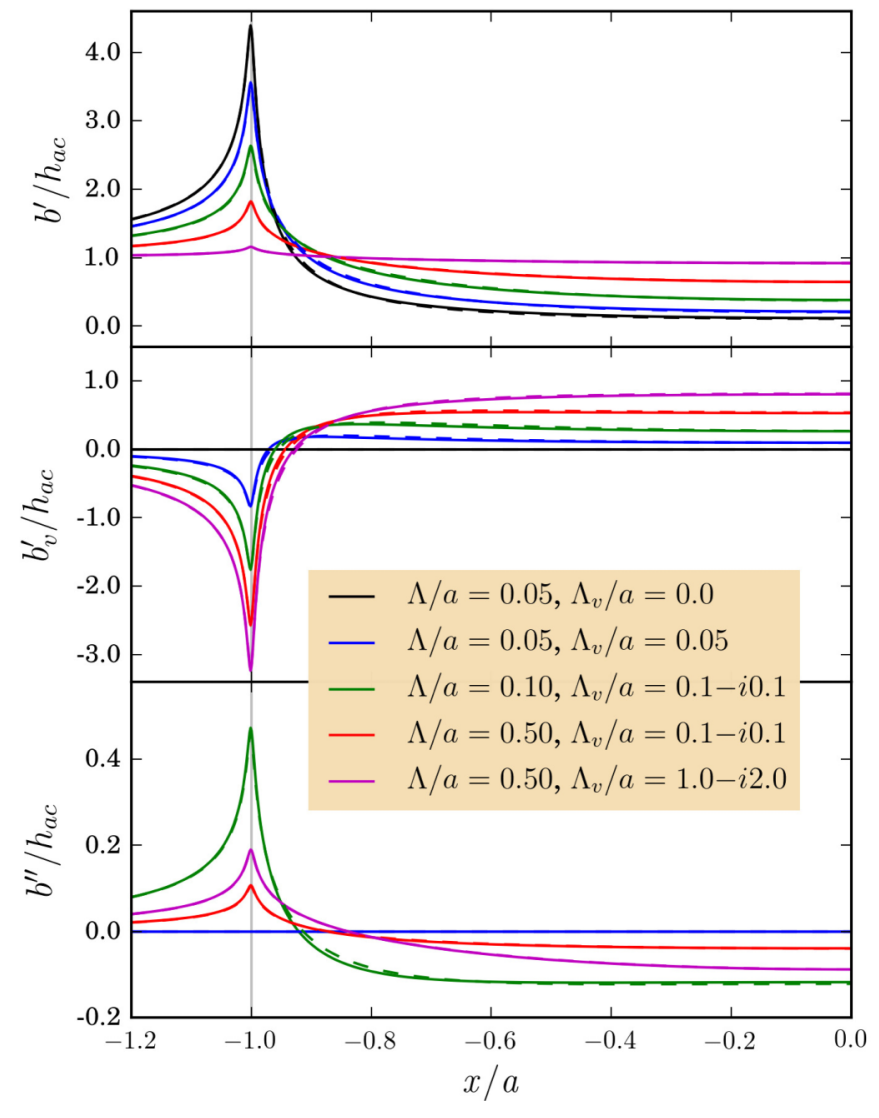

FIG. 1. Profiles of the in-phase (top) and out-of-phase (bottom) components of the ac magnetic permeability, $b_{a c}(x) / h_{a c}$ (in units of $\mu_{0}$ ) calculated using a full numerical inversion of Eq. (7) (dashes) and the analytic approximation Eq. (8) (full lines). The middle panel presents similar profiles for the vortex contribution to $b^{\prime} / h_{a c}$ (see text).

Exact analytical solutions to Eq. (7) are only available for the limiting cases $\Lambda=0$ and $\Lambda \rightarrow \infty$ [26]. Therefore, in general, $J(x)$ is to be determined by numerical inversion of Eq. (7), which can be computationally demanding. However, an empirical interpolation formula proposed by Vodolazov and co-workers [32,33] provides a very useful approximate solution for the dc version of Eq. (7) [i.e., for Eq. (1) with $B_{v}=$ $0]$ which covers a wide range of the superconducting strip parameters. We have found (cf. below) that this approximate solution for the dc Meissner sheet current, which is a real function of real $\Lambda$, can be extended to the complex $\Lambda_{a c}$ plane and is also an excellent approximation for the complex ac sheet current, that is

$$
J_{a c}(x, \omega) \simeq \frac{h_{a c} x}{\sqrt{\alpha\left(a^{2}-x^{2}\right)+2 \beta a \Lambda_{a c}(\omega)}},
$$

where the empirical parameters $\alpha=1 / 4-0.45\left(\Lambda_{a c} / a\right)^{0.5}+$ $0.69\left(\Lambda_{a c} / a\right)^{0.8}$ and $\beta=1 / 2 \pi+\Lambda_{a c} / 2 a$ are the same obtained in $[32,33]$, but with $\Lambda$ replaced by its complex counterpart $\Lambda_{a c}$.

In Fig. 1, we present the profiles of the local magnetic permeability $b_{a c}(x) / h_{a c}$ of a superconducting strip, which is the typical quantity accessed in ac susceptibility imaging experiments, using either scanning Hall probes or
SQUIDs $[3,8,9,34]$. This quantity was calculated for different values of $\Lambda$ and $\Lambda_{v}$, covering $0.05 a \leqslant\left|\Lambda_{a c}\right| \leqslant 2.5 a$, at a height $z_{0}=0.005 a$ above the plane of the strip. This value for $z_{0}$ is close to the typical probe height used in our experiments [35]. The real $\left(b^{\prime}\right)$ and imaginary $\left(b^{\prime \prime}\right)$ parts of $b_{a c}(x)$ were obtained by numerically integrating Ampère's law,

$$
b_{a c}\left(x, z_{0}\right)=\mu_{0} h_{a c}+\frac{\mu_{0}}{2 \pi} \int_{-a}^{a} \frac{\left(x-x^{\prime}\right) J_{a c}\left(x^{\prime}\right)}{\left(x-x^{\prime}\right)^{2}+z_{0}^{2}} d x^{\prime},
$$

and using the sheet current calculated numerically from Eq. (7) (dashes) and its analytical approximation, Eq. (8) (full lines). It is clear that, within a broad range of scenarios for the ac screening length, Eq. (8) provides an excellent approximation to the linear ac response of a superconducting (or metallic, for that matter) strip.

It is instructive to go further and analyze separately the contribution of vortices to the ac response, $b_{v}$. This can be accomplished easily by noticing that $b_{a c}=b_{M}+b_{v}$, where $b_{M}$ is the response of the Meissner screening currents, which can be obtained by setting $\Lambda_{v}=0$ in Eq. (9) (that is, by ignoring vortex motion). Since $b_{M}$ is purely inductive (real), we have

$$
b_{v}=b_{v}^{\prime}+i b_{v}^{\prime \prime}=\left(b^{\prime}-b_{M}\right)+i b^{\prime \prime} .
$$

The profiles of $b_{v}^{\prime}$ and $b_{v}^{\prime \prime}=b^{\prime \prime}$ are depicted in the middle and bottom panels of Fig. 1, respectively. In particular, in the regions near the sample edge, $b_{v}^{\prime}$ and $b_{v}^{\prime \prime}$ are very sensitive to the specific value of $\Lambda_{v}$. Typically $b_{v}^{\prime}\left(b_{v}^{\prime \prime}\right)$ presents a strong negative (positive) signal near the sample edge that changes to positive (negative) when entering the superconductor from outside in. This suggests that imaging experiments performed in this area can reveal valuable information regarding the vortex dynamic in the sample.

Equation (7) and its approximate solution, Eq. (8), provide a general picture of the macroscopic response of thin, wide strips of any type-II material as long as the linear approximation can be used. Now the problem is reduced to finding the complex ac screening length $\Lambda_{a c}$, which is associated with the vortex dynamical response function through Eq. (5).

\section{B. Response function of a nanostructured superconducting strip}

We consider a superconducting strip with a square array of artificial pinning centers regularly spaced by the lattice constant $a_{p}$. We assume the artificial traps saturate above one flux quantum per trap, in a way that above the first matching field $B_{1}=\phi_{0} / a_{p}^{2}$ (where $a_{p}$ is the lattice constant of the square pinning array) vortices not fitting the traps occupy the interstitial positions.

In order to estimate the response function and thereby the ac penetration depth of this nanostructured superconducting film, we use the two-species model introduced in Ref. [24]. In short, this model considers the vortex lattice as comprising a sublattice of vortices trapped in the antidots and a sublattice of interstitial vortices. Both sublattices are assumed to move rigidly and are connected elastically to each other via vortexvortex interactions. This is a good approximation at the first and second matching fields, where the sublattices are, typically, square lattices and vortex-vortex interactions within each sublattice cancels out. This is not the case at nonmatching fields. However, we keep this approximation in the whole 


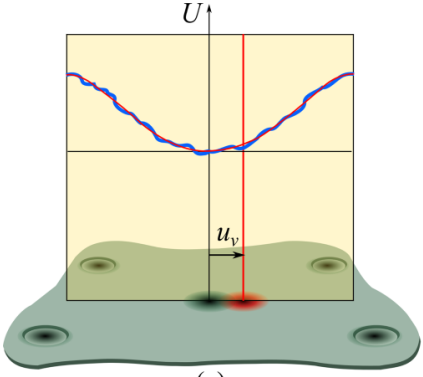

(a)

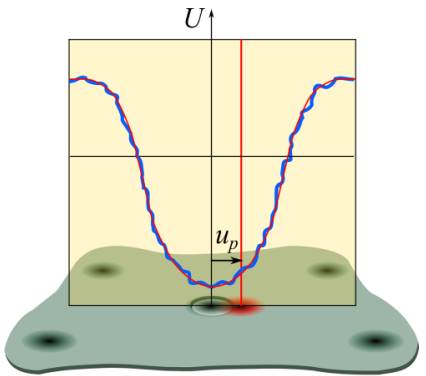

(b)
FIG. 2. Schematic representation of the potential energies of (a) an interstitial vortex caged by artificially pinned vortices and (b) a vortex trapped in an artificial pinning center near the second matching field. In both panels, the blue (red) curve represents the potential energy with (without) the contribution from natural defects. Cartoons: gray (red)-shaded disks represent vortices in their (out-of-)equilibrium position.

$0<B<2 B_{1}$ field range in the expectation that the dynamical response at nonmatching fields will be captured at least qualitatively.

Within the two-species model, the interstitial vortices are subjected to a cage potential induced by the pinned vortices, while the pinned vortices probe in turn a potential energy resulting from the superposition of the artificial pinning potential and the cage induced by the interstitial vortices (see, for instance, Refs. [12,24] for more details). A schematic representation of the potential energies of each vortex species is depicted in Fig. 2 (thin red curves).

In what follows, we will extend the two-species model in order to include explicitly the effect of natural sample inhomogeneities. These induce a random pinning potential that superimposes to the potential energies considered in the original two-species model. Cartoons of the total energy potentials are depicted in Fig. 2 as the thick blue lines. The typical barriers, $U_{0}$, of the random pinning landscape are expected to be small compared to the cage potential. However, the restoring force of a potential well near its local minimum is determined by its curvature, roughly given by $U_{0} / r^{2}$, where $r$ the characteristic well size. For the cage potential, $r \sim a_{p}$, whereas for a sample inhomogeneity $r \sim \xi$, which is typically much smaller than $a_{p}$. Therefore, the typical spring constant due to sample inhomogeneities can be considerably stronger than the cage spring constant. Moreover, because the energy barriers are typically small, thermal activation through the randomly distributed pinning centers can play an important role in the vortex dynamics.

Within the linear approximation, the equations of motion for the local displacement field of the pinned vortex sublattice $\left(u_{p}\right)$ and the interstitial vortex sublattice $\left(u_{i}\right)$ can be written as [36]

$$
\begin{gathered}
\eta \dot{u}_{p}=-\alpha_{p} u_{p}-\alpha_{r} u_{p}-(n-1) \alpha_{v}\left(u_{p}-u_{i}\right)+\phi_{0} J \\
\eta \dot{u}_{i}=-\alpha_{r} u_{i}+\alpha_{v}\left(u_{p}-u_{i}\right)+\phi_{0} J
\end{gathered}
$$

where $n=B / B_{1}$ is the occupation number, $\alpha_{p}$ is the spring constant representing the interaction between a pinned vortex and an artificial pinning center, $\alpha_{v}$ is the spring constant due to the cage potential, and $\alpha_{r}$ represents the effect of weak random pinning. Following Brandt [30], $\alpha_{r}$ is a time-dependent relaxing elastic coefficient which accounts for thermal activation processes over the random pinning landscape. In the frequency domain, it is given by

$$
\alpha_{r}(\omega)=\frac{\alpha_{r} 0}{1-i / \omega \tau},
$$

where $\alpha_{r 0}$ is the typical elastic constant of the weak pinning centers, $\tau \sim\left(\eta / \alpha_{r 0}\right) e^{U_{0} / k_{B} T}$ is the hopping time, and $U_{0}$ is the typical energy barrier.

For the case of a small, low-frequency ac excitation, $J=$ $J_{a c}(x) e^{i \omega t}\left(\eta \omega \ll \alpha_{p}, \alpha_{v}, \alpha_{r 0}\right)$, one can neglect the flux-flow term in Eqs. (11) and (12). Within linear approximation, $u_{p}(x, t)=u_{p}(x, \omega) e^{i \omega t}$ and $u_{i}(x, t)=u_{i}(x, \omega) e^{i \omega t}$, this leads to the following solution for the pinned and interstitial vortex displacement fields:

$$
\begin{aligned}
& u_{p}(x, \omega)=\frac{\left(n \alpha_{v}+\alpha_{r}\right) \phi_{0} J_{a c}(x)}{\alpha_{p}\left(\alpha_{v}+\alpha_{r}\right)+\alpha_{r}\left(n \alpha_{v}+\alpha_{r}\right)}, \\
& u_{i}(x, \omega)=\frac{\left(\alpha_{p}+n \alpha_{v}+\alpha_{r}\right) \phi_{0} J_{a c}(x)}{\alpha_{p}\left(\alpha_{v}+\alpha_{r}\right)+\alpha_{r}\left(n \alpha_{v}+\alpha_{r}\right)},
\end{aligned}
$$

where the frequency dependence of $\alpha_{r}$ is implicit.

The response function can now be obtained by evaluating the local center-of-mass displacement field, that is, $u(\omega)=$ $\frac{1}{n}\left[u_{p}+(n-1) u_{i}\right]$. From Eqs. (14) and (15):

$$
\mu_{v}(\omega)=\phi_{0} \frac{\left(1-\frac{1}{n}\right) \alpha_{p}+n \alpha_{v}+\alpha_{r}}{\alpha_{p}\left(\alpha_{v}+\alpha_{r}\right)+\alpha_{r}\left(n \alpha_{v}+\alpha_{r}\right)} .
$$

Notice that from the linearity condition implicit in Eq. (3) the position dependence of $\mu_{v}$ cancels out. Of course, this relies on the assumption that the coupling constants are themselves not position dependent, which is a reasonable approximation for uniform flux distribution.

It is also worth mentioning that, by taking $\alpha_{p}, \alpha_{v}=0$, one recovers the single-species models, in which the whole dynamics is described in terms of a single elastic constant. Accordingly, in the low-frequency limit considered here one would have the well known Campbell response [37], $\mu_{v}(\omega)=$ $\phi_{0} / \alpha_{r}(\omega)$, but with a dispersive, complex Labusch constant, given by Eq. (13), accounting for linear flux creep.

\section{SCANNING HALL PROBE EXPERIMENTS}

\section{A. Sample layout and equilibrium vortex configurations}

The sample under investigation is a nanostructured highquality $\mathrm{Pb}$ superconducting strip of width $2 a=600 \mu \mathrm{m}$, and thickness $d=50 \mathrm{~nm}$; see Fig. 3(a). The sample contains a square array of square antidots with an antidot void area of $b^{2}=0.6 \times 0.6 \mu \mathrm{m}^{2}$, as obtained by electron-beam lithography and subsequent lift-off. The periodicity of the antidot lattice is $a_{p}=3 \mu \mathrm{m}$. Thereby, the magnetic flux density at which the number of antidots coincides with the number of vortices is $B_{1}=\phi_{0} / a_{p}^{2}=0.2298 \mathrm{mT}$. This particular sample layout is chosen as it is well studied in literature by macroscopic ac-susceptibility measurements [15,38].

The sample was deposited on top of a $\mathrm{SiO}_{2}$ insulating substrate and covered by a Ge buffer layer of $60 \mathrm{~nm}$ to prevent oxidation. An additional gold layer of $50 \mathrm{~nm}$ covers the whole strip and allows one to approach the sample surface 


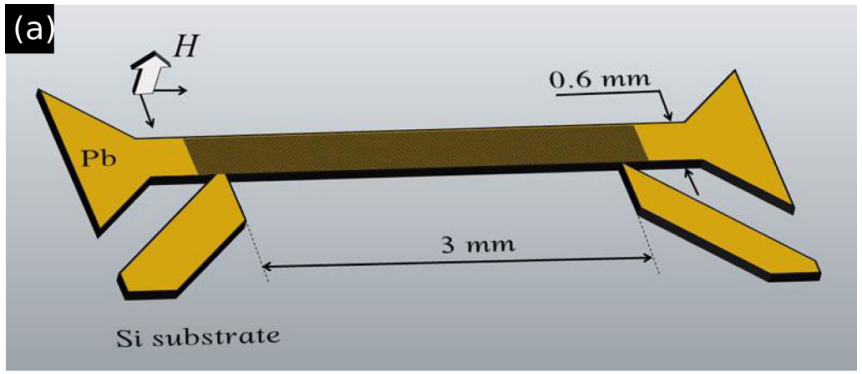

(b)

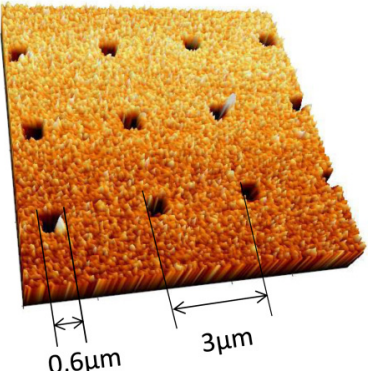

FIG. 3. (a) Schematic layout of the investigated transport bridge with a patterned area. Most of the scanning Hall probe microscopy images were obtained in a $16 \times 16 \mu \mathrm{m}^{2}$ area at the border of the sample. The dc and ac magnetic fields are applied perpendicularly to the plane of the $\mathrm{Pb}$ film. (b) Atomic force microscopy image of the sample surface.

in STM mode. Figure 3(b) shows a representative atomic force microscopy image of the sample surface. In all cases the magnetic field (ac and dc) is applied perpendicular to the plane of the film. The superconductor to normal transition at zero field occurs at $T_{c}=7.2 \mathrm{~K}$ as measured by monitoring the in-phase and out-of-phase response to an applied ac magnetic field with the Hall probe while sweeping the temperature through the normal metal-to-superconductor transition.

We start by investigating the vortex distributions at $T=$ $4.2 \mathrm{~K}$ generated after field cooling (FC) at a given value of the applied dc magnetic field, $H_{d c}$. This procedure guarantees uniform flux distribution (with macroscopic flux density $B \simeq$ $\mu_{0} H_{d c}$ ) and thereby allows us to explore near to equilibrium vortex states, which are established at a temperature $T_{f}$ close to $T_{c}$ [39]. The corresponding vortex configuration is revealed by measuring the local (microscopic) magnetic induction, $b_{z}(x, y)$, in a scan range of $16 \times 16 \mu \mathrm{m}^{2}$ with a submicro-sized Hall probe $\left(0.5 \times 0.5 \mu \mathrm{m}^{2}\right)$, approximately $1.2 \mu \mathrm{m}$ above the sample surface. The mapping of $b_{z}(x, y)$ was obtained using a modified low-temperature SHPM from Nanomagnetics Instruments [40]. The above described procedure is followed for field values between $H_{d c}= \pm 3.9 H_{1}$ (with $H_{1}=B_{1} / \mu_{0}$ ) in steps of $\Delta H_{d c}=+0.004 \mathrm{mT} / \mu_{0}$, which is less than the field necessary to have one additional vortex per scan area $\phi_{0} /\left(16 \times 16 \mu \mathrm{m}^{2}\right)=0.008 \mathrm{mT}$. Some selected experimental results are shown in Figs. 4 and 5 for fields respectively below and above the first matching field. The color scale is adjusted for every image to maximize the contrast.

It is clear from Figs. 4 and 5 that the sample shows commensurability effects. Not only first matching is nicely present; also fractional matching features can be identified in agreement with previous theoretical predictions [41] (shown schematically in Fig. 4 for comparison). However, the com-
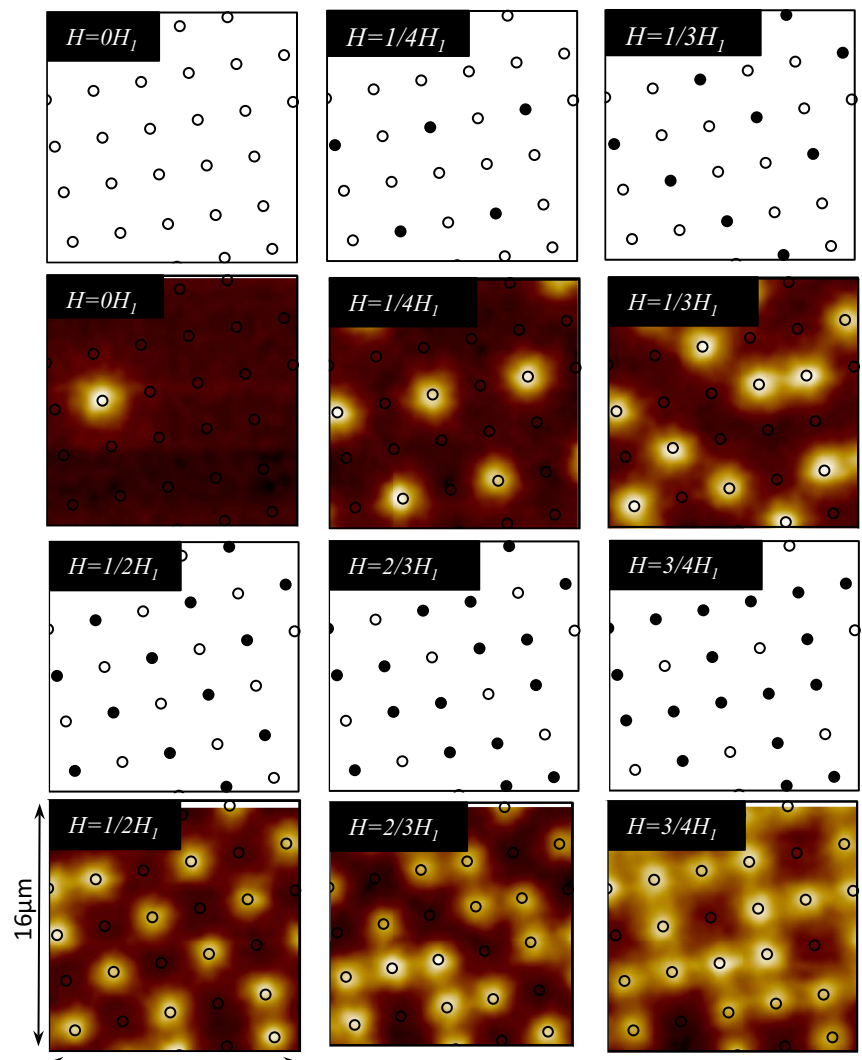
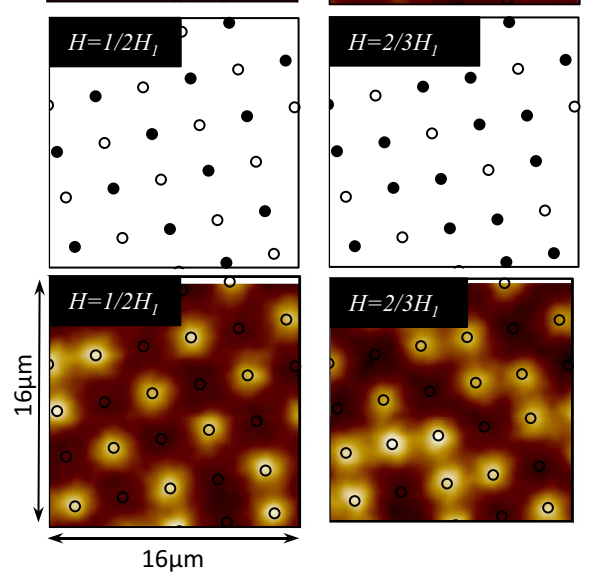

FIG. 4. Contour plots: scanning Hall probe microscopy images obtained after field cooling down to $T=4.2 \mathrm{~K}$ in presence of the indicated dc magnetic field $H=0 H_{1}, 1 / 4 H_{1}, 1 / 3 H_{1}, 1 / 2 H_{1}, 2 / 3 H_{1}$, and $H=3 / 4 H_{1}$. The open circles represent the antidot positions. The theoretical prediction of Ref. [41] is shown schematically above the respective image, with filled circles representing the antidots occupied by a singly quantized vortex.

mensurate vortex distributions at fractional matching fields present a considerable amount of structural defects, possibly reminiscent of domain formation [42,43]. Just above the first matching field, the first interstitial vortices appear, while upon further increasing the dc field a combination of interstitial and double quantized vortices (at the pinning sites) is established. This suggests that the saturation number $n_{s}$ of the antidots at the freezing temperature is $1<n_{s}<2$.
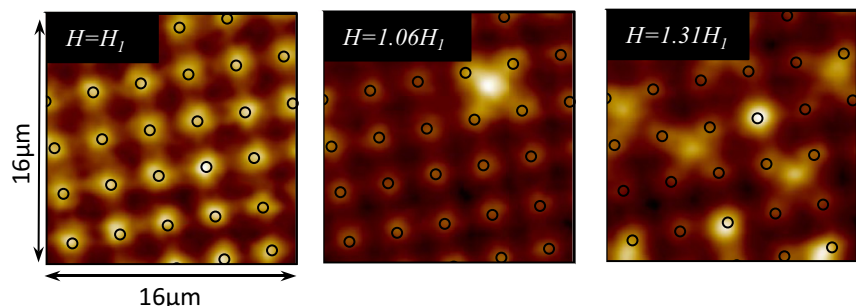

FIG. 5. Scanning Hall probe microscopy images obtained after field cooling down to $T=4.2 \mathrm{~K}$. From left to right, for $H=$ $H_{1}, 1.06 H_{1}$ and $H=1.31 H_{1}$, respectively. The open circles indicate the position of the antidots. 


\section{B. Scanning ac susceptibility images}

We track the local ac response of the different nearequilibrium vortex configurations obtained after the FC process described in Sec. III B using the scanning ac susceptibility microscopy (SSM) technique. The details of the technique can be found in Ref. [3]. In summary, the superconducting sample is surrounded by a copper coil, which is mounted coaxially in the bore of the superconducting magnet and generates an ac field $h(t)=h_{a c} \cos \omega t$ parallel to the dc field. In all measurements we used $\mu_{0} h_{a c}=0.016 \mathrm{mT}$. The time dependent local induction, $b_{z}(\vec{r}, t)$, with $\vec{r}=\left(x, y, z_{0}\right)$, is picked up by the Hall probe at a height $z_{0}=1.5 \mu \mathrm{m}$ above the sample surface, and analyzed by a lock-in amplifier using as a reference the applied ac magnetic field. The mapped firstorder in-phase, $b^{\prime}(x, y)$, and out-of-phase, $b^{\prime \prime}(x, y)$, Fourier components provide us with a first (linear) approximation to the local in-phase (inductive) and out-of-phase (dissipative) responses, respectively. The zeroth order (dc) component, $b_{d c}(x, y)$, maps the time averaged flux distribution. To avoid unwanted effects such as eddy current heating, the skin effect of the sample holder, or the frequency dependence of the Hall probe sensitivity, we performed all measurements at a fixed low driving frequency of $f=\omega / 2 \pi=77.123 \mathrm{~Hz}$. The dwell time at every pixel $\left(\tau_{\text {pix }}\right)$ and the integration time of the lock-in $\left(\tau_{\text {int }}\right)$ are chosen appropriately $\left(\tau_{\text {pix }}, \tau_{\text {int }} \gg 1 / f\right)$ and the measured phase between the picked up signal and the ac magnetic field drive is fixed above $T_{c}=7.2 \mathrm{~K}$ to zero.

Figure 6 shows the maps of $b_{d c}(x, y)$ (first row), $b^{\prime}(x, y)$, and $b^{\prime \prime}(x, y)$ acquired following the above described procedure for a few selected values of $H_{d c}$ at the temperatures $T=6.7 \mathrm{~K}$ $\left(T / T_{c}=0.931\right)$ and $T=6.9 \mathrm{~K}\left(T / T_{c}=0.958\right)$ in a fixed area which includes the sample edge (represented in the images by the white lines). The estimated positions of the antidots are shown in order to help distinguish between trapped and interstitial vortices. In order to isolate the vortex contribution to the sample response, $b_{v}(x, y)$, we have also subtracted the pure Meissner response, which corresponds to the in-phase response at $H_{d c}=0$, following Eq. (10). Therefore, the third row represents the in-phase component of $b_{v}$. Since the Meissner response is purely real, the fourth row, $b^{\prime \prime}(x, y)$, also represent the out-of-phase component of the vortex contribution. For a better visualization, we reduced noise in all images using a $\sigma=2$ Gaussian smoothing. The images in rows 2 to 4 are in units of $\mu_{0} h_{a c}$, so the numerical values depicted in the color bars represent the local magnetic permeability.
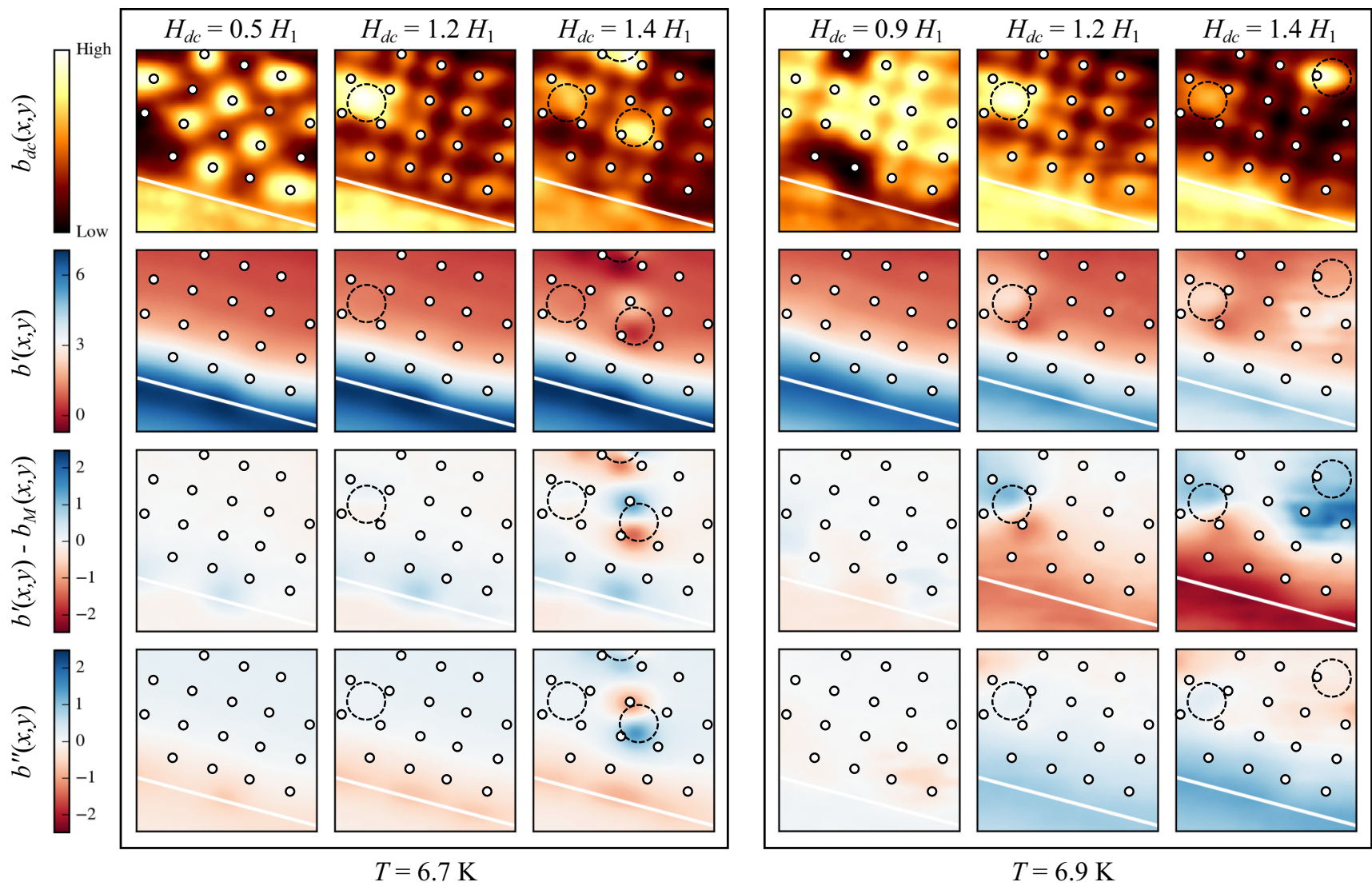

FIG. 6. SSM images showing the ac response (mapped in a $16 \times 16 \mu \mathrm{m}^{2}$ region near the sample edge) to a $77.123 \mathrm{~Hz}$ excitation field of amplitude $\mu_{0} h_{a c}=0.016 \mathrm{mT}$ for different field values at $T=6.7 \mathrm{~K}$ (left) and $T=6.9 \mathrm{~K}$ (right). The first row shows the dc (time-average) flux distributions. The in-phase and out-of-phase components of the total ac response are mapped in the second and fourth rows, respectively. The in-phase vortex response, defined as the difference between the in-phase and the Meissner responses, is shown in the third row. In all images, the white dots and the white line show schematically the position of the square antidots and the sample edge, respectively. The dashed circles highlight the position of selected interstitial vortices. All red-blue color bars are in units of $\mu_{0} h_{a c}$. 
In contrast to the images acquired away from the sample edge (Figs. 4 and 5), the dc images shown in the first row of Fig. 6 reveal that the number of vortices near the sample edge is in general smaller than that expected for the external dc field value. This is consistent with molecular dynamics simulations of vortices in a superconducting slab with periodic pinning, which predicted that, for a wide range of applied field values, vortices distribute uniformly over the bulk of the sample, while near the sample surface the vortex density depletes considerably [44]. Such configurations stem from the interplay between the pining potential and the Bean-Livingston (BL) barrier [45], which tends to keep vortices away from the sample surface within a distance of the order of $\lambda[46,47]$. In the present case, since the field is applied perpendicular to the sample, the width of the flux-depleted region is at least of the order of $\Lambda=\lambda^{2} / d$. Additional depletion can also be attributed to the long range nature of the screening current $[48,49]$. However, the experimental observation in antidot systems of pronounced matching effects at values of the applied field that coincide with the expected flux density, $\mu_{0} H=n B_{1}$ [10-22], suggests that the depletion zone is much narrower than the sample itself. In any case, as shown in the Supplemental Material [31], our results do not depend strongly on the width of the flux-depleted region.

Now we turn our attention to the ac response. We first note, at $6.7 \mathrm{~K}$, a pronounced enhancement of the inductive response near the sample edge, which is the hallmark of the ac Meissner effect in strips, as discussed in Sec. II A. Such enhancement seems not to fade upon increasing the dc field. However, for $H_{d c}>1.4 H_{1}$, localized responses induced by the oscillation of a few interstitial vortices could be observed superimposed to the screening profile. The motion of these vortices can be better appreciated in the images of the in-phase and out-of-phase vortex responses (third and fourth rows, respectively), where the typical peak-valley pair profile, centered at the mean vortex position, reveals the direction of motion [3,9]. The direction of motion is not exactly perpendicular to the sample border, because it is also influenced by the asymmetries in the local energy landscape probed by the vortex $[9,34]$. Since both components of the local permeability nearby the moving vortices are typically of the same order of magnitude, their motion is clearly phase lagged with respect to the ac excitation. If we take into account the very small frequency used in the experiment, this is a clear evidence of thermally activated hopping of these vortices. In addition, small permeability peaks at the sample edge can also be identified. We believe these are small nonlinear effects produced by vortices nucleating and annihilating at weak spots of the sample edge as the excitation field is cycled. Apart from these isolated activities, the profiles of the vortex response at $6.7 \mathrm{~K}$ are essentially flat for all investigated dc field values, indicating that, in average, vortices are strongly pinned and contribute negligibly to the picked up ac response. Therefore, at this temperature, $\Lambda_{a c} \simeq \lambda^{2} / d$ and the strip responds to the ac excitation as if in the Meissner state.

As opposed to the $T=6.7 \mathrm{~K}$ case discussed above, at $6.9 \mathrm{~K}$ the Meissner screening diminishes considerably upon increasing $H_{d c}$ above the first matching field. While for $H_{d c} \leqslant H_{1}$ the vortex response contributes negligibly to the overall sample response (as illustrated by the flat profiles of $b_{v}^{\prime}$ and $b^{\prime \prime}$ for the $H=H_{1}$ case), for $H_{d c}>H_{1}$ both in-phase and out-of-phase components of $b_{v}$ reveal a strong, delocalized contribution of vortices to the sample ac permeability and ac penetration depth. Some localized vortex activities can be observed but this time they are superimposed to a background permeability profile that resembles those of Fig. 1, that is, $b_{v}^{\prime}$ $\left(b_{v}^{\prime \prime}\right)$ is strongly negative (positive) nearby the sample edge and smoothly crosses over to positive (negative) values as crossing the sample from outside in. This is an evidence that vortex dynamics contribute strongly to the macroscopic sample response. Moreover, this contribution is highly dissipative, indicating that for $T=6.9 \mathrm{~K}$ the mean vortex dynamics in the sample is dominated by thermal activation processes.

It is important to mention that, due to the strong nonlocality of the field-current relation in a thin strip under perpendicular field, the vortex permeability profiles observed in the scan area build up with the contribution of moving vortices over the whole sample. For instance, for $H_{1}<H<1.2 H_{1}$, no interstitial vortices were observed within the scan area and no feature characteristic of single moving vortices was observed. However, permeability profiles very similar to those shown in Fig. 6 for $H_{d c}=1.2 H_{1}$ can still be observed, which is a result of the dynamical response of interstitial vortices that do populate the bulk of the sample. The fact that the SSM technique allows us to resolve this collective response at scales smaller than $\left|\Lambda_{a c}\right|$ provides us with a unique opportunity to confront the theoretical predictions of Sec. II and extract details about the vortex dynamics in the nanostructured superconducting strip.

\section{MODEL VS EXPERIMENT}

To compare our experimental results to the model developed in Sec. II, we first establish estimates of the different elastic constants. The cage constant has been calculated exactly for an infinite square array of artificial pinning centers with period $a_{p} \ll \pi \Lambda$ and perfectly ordered vortex configurations [24]: $\alpha_{v}=\pi \epsilon / a_{p}^{2}$, where $\epsilon=\phi_{0}^{2} / 2 \pi \mu_{0} \Lambda$ is the energy scale. For a narrow strip, however, the infinite sum over trapped vortices is obviously an overestimate. Moreover, disorder in the vortex configuration softens $\alpha_{v}$ even further. Therefore, we assume

$$
\alpha_{v}=c_{v} \frac{\pi \epsilon}{a_{p}^{2}},
$$

where $c_{v}$ (typically $<1$ ) is an empirical prefactor, which we will further assume to be temperature independent.

For an antidot with radius $R \lesssim \sqrt{2} \xi$, a lower estimate of the pinning potential can be obtained by calculating the gain in condensation energy as a vortex approaches the antidot [1]: $U_{A D}(r) \simeq U_{A D}^{(0)} /\left(1+r^{2} / 2 \xi^{2}\right)$, with $U_{A D}^{(0)}=(R / 2 \xi)^{2} \epsilon / 2 . \alpha_{p}$ follows immediately from the second derivative of $U_{A D}(r)$ :

$$
\alpha_{p} \simeq\left(\frac{R}{2 \xi}\right)^{2} \frac{\epsilon}{\xi^{2}} .
$$

For an antidot system with $n_{s}=1$, the prefactor $(R / 2 \xi)^{2}$ is of the order 1 . For a weak pinning center, such as a material defect, the potential well can be modeled in a similar way, $U_{p}(r) \simeq U_{0} /\left(1+r^{2} / 2 \xi^{2}\right)$, with $U_{0}=c_{r} \epsilon / 2$, but with a prefactor $c_{r}$ considerably smaller. Therefore, the typical 
spring constant of weak pinning centers can be estimated as

$$
\alpha_{r 0} \simeq c_{r} \frac{\epsilon}{\xi^{2}}
$$

and the hopping time appearing in Eq. (13) can be expressed as

$$
\tau \sim \frac{\eta}{\alpha_{r 0}} \exp \left(\frac{c_{r} \epsilon}{2 k_{B} T}\right),
$$

with $c_{r} \ll 1$ typically.

Before proceeding with the comparison between the model and the experimental results for nonzero dc fields, we fitted Eq. (8) to the experimental data for $H_{d c}=0$. In this case (ac Meissner state) no vortices are present and the ac perturbation is expected to be screened within a length $\Lambda_{a c}=\lambda^{2} / d$. We performed the fitting for both temperatures using the zero temperature penetration depth, $\lambda(0)$, and the probe height, $z_{0}$ [appearing in Eq. (9)] as free parameters and adopting the Ginzburg-Landau scaling for $\lambda(T)$. The best fit was obtained for $\lambda(0)=65 \mathrm{~nm}$ and $z_{0}=1.38 \mu \mathrm{m}$. These values are in agreement with those obtained in Ref. [35] by analysis of the permeability profiles of the dc Meissner state [50]. The fitting results are discussed in more detail in the Supplemental Material [31].

With the values for $z_{0}$ and $\lambda(0)$ at hand, and using $\xi(0)=$ $35 \mathrm{~nm}$, estimated from the temperature dependence of the upper critical field, we take $c_{v}$ and $c_{r}$ as the only free empirical parameters to be determined for nonzero dc fields. The fitting procedure is as follows. For each temperature and in the field range $0 \leqslant H_{d c} \leqslant 2 H_{1}$ we integrate both real and imaginary components of the acquired magnetic response over the whole scan area in order to obtain the inductive and dissipative components of the mean magnetic permeability, $\left\langle b^{\prime}\right\rangle / h_{a c}$ and $\left\langle b^{\prime \prime}\right\rangle / h_{a c}$, respectively, as functions of $H_{d c}$. Since the scan area encloses the sample edge and adjacencies, these averages are highly sensitive to the specific value of $\Lambda_{v}$ and, thereby, on the vortex dynamics within the strip, as discussed in Sec. II A. Then, we used Eqs. (8) and (9) to compute the theoretical local permeability and averaged it over an area equivalent to that used in the experiment. The fitting parameters $c_{v}$ and $c_{r}$ were included in the calculation via the theoretical expression for the ac penetration depth, $\Lambda_{a c}=\Lambda+\Lambda_{v}$, with $\Lambda_{v}$ given by Eqs. (5) and (16). For $0 \leqslant H_{d c} \leqslant H_{1}$, in which case interstitials are absent, we used the response function given by Eq. (14) with $\alpha_{v}$ set to zero.

In Figs. 7(a) and 7(b), we present the results (for $T=6.7 \mathrm{~K}$ and $6.9 \mathrm{~K}$, respectively) of integrating the inductive component of the sample response only in the direction along the strip. The data is organized as contour plots in the plane defined by the perpendicular position with respect to the sample edge and $H_{d c}$. This allows for a better visualization of how the permeability profiles evolve when increasing the dc field. In Figs. 7(e) and 7(f), we present the result of integrating over the whole scan area (symbols). In both cases, we used the raw data acquired by the SSM system. No smoothing procedure was used. It is clear that, for $T=6.7 \mathrm{~K}$, the field penetration is almost field independent within the investigated field range, although some spreading of the penetrating flux can be identified above $H_{1}$. Notice that the data becomes more noisy for $1.2 H_{1} \lesssim H_{d c} \leqslant 2 H_{1}$, which is a result of the motion of a few interstitial vortices within the scan area as discussed in the previous section. At $T=6.9 \mathrm{~K}$, the flux penetration is essentially field independent for $H_{d c} \leqslant H_{1}$ but
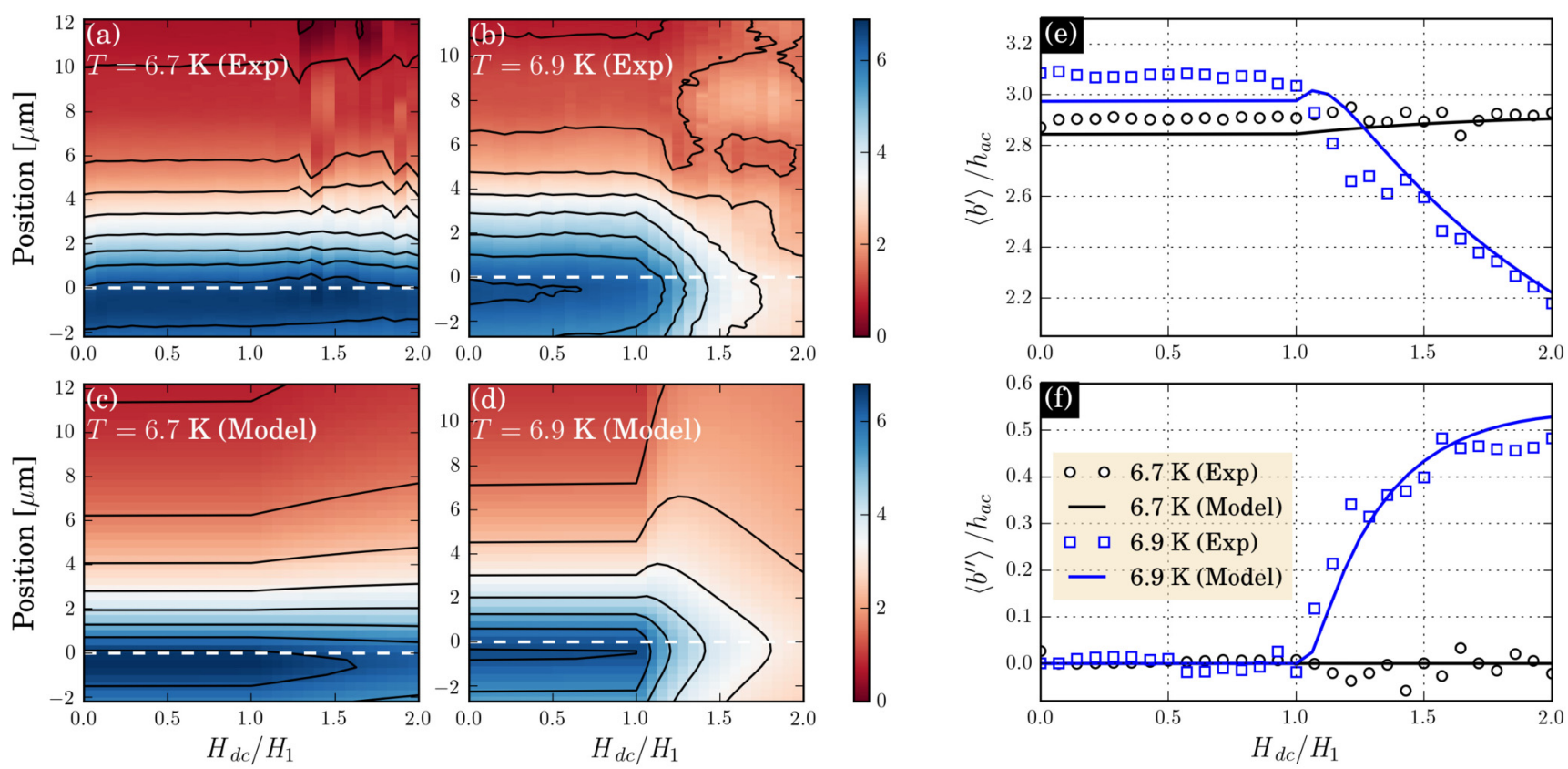

FIG. 7. Contour plots of the inductive magnetic permeability, $b^{\prime} / h_{a c}$ (in units of $\mu_{0}$ ), measured at (a) $T=6.7 \mathrm{~K}$ and (b) $T=6.9 \mathrm{~K}$ and averaged over the direction along the strip, as a function of position across the strip and the applied dc field (in units of $H_{1}$ ). Panels (c) and (d) correspond to similar contour plots calculated using the model described in Sec. II and the values of the empirical parameters $c_{v}$ and $c_{r}$ appearing in Eqs. (17)-(20). These values were obtained by fitting the model (lines) to the experimental data (symbols) corresponding to averaging the in-phase (e) and the out-of-phase (f) SSM images over the scan area. 


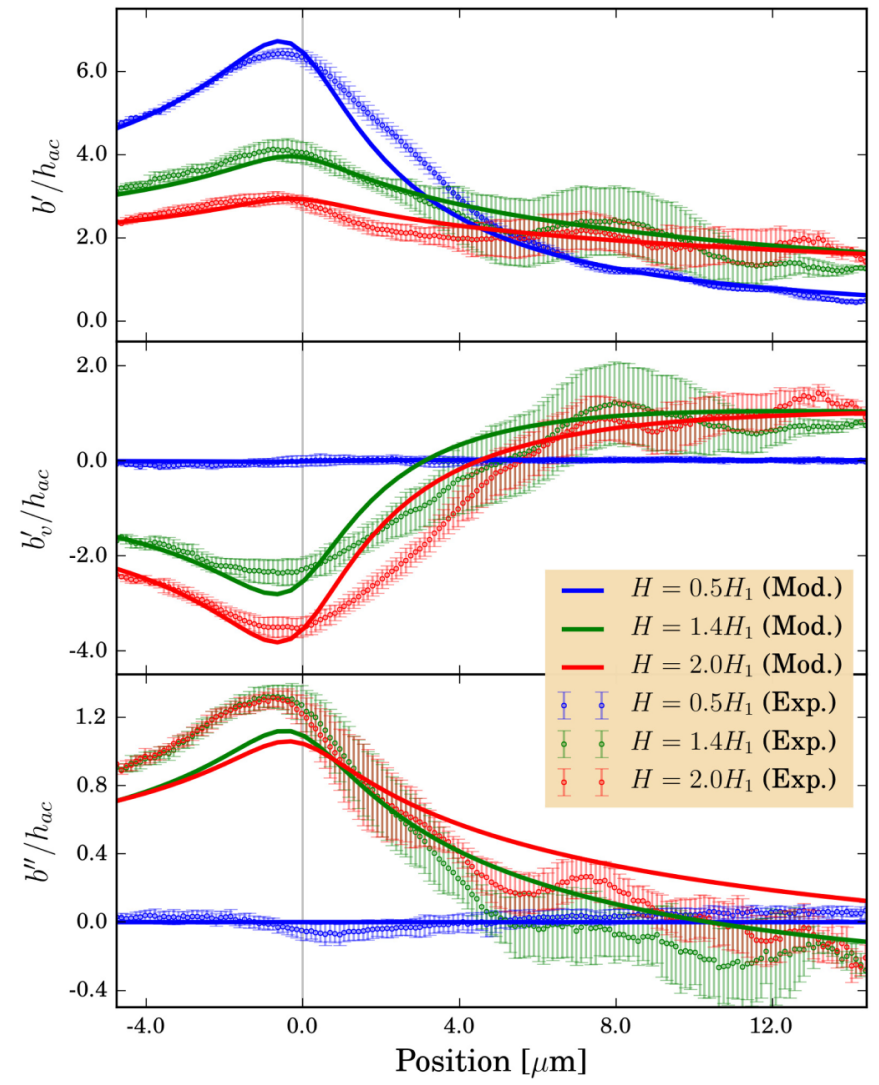

FIG. 8. Symbols: experimental cross-section profiles of the permeability averaged over lines parallel to the strip at $6.9 \mathrm{~K}$ for selected dc field values. Error bars are standard deviations from the mean. Lines: theoretical permeability profiles calculated using the fitting parameters $c_{v}=0.09$ and $c_{r}=0.019$.

changes remarkably to a much smoother profile precisely at $H_{d c}=H_{1}$, as can also be observed in the experimental $\left\langle b^{\prime}\right\rangle\left(H_{d c}\right)$ and $\left\langle b^{\prime \prime}\right\rangle\left(H_{d c}\right)$ curves. As a result, a strong reduction in the inductive permeability integrated over the scan area can be observed. In addition, the dissipative permeability, which is zero below $H_{1}$, becomes positive for $H_{1} \lesssim H_{d c} \leqslant 2 H_{1}$, signaling that thermal hopping effects are triggered just above $H_{1}$, that is, as soon as the first interstitials populate the sample.

After exploring a wide range of values of the fitting parameters, we have found that $c_{v}=0.09$ and $c_{r}=0.019$ give the best fit to the experimental data. As can be observed in Figs. 7(e) and 7(f), the model (full lines) is capable of fitting reasonably well and simultaneously the inductive and dissipative components of the sample response collected in the whole field range, for both temperatures. The agreement between the theoretical and experimental cross-section profiles can be appreciated in Fig. 8, where we show the components of the permeability for $T=6.9 \mathrm{~K}$ and a few selected dc field values. In particular, for $H=0.5 H_{1}$, while the agreement is excellent deeply inside and outside the sample, one can observe a mismatch between the experimental data and the model near the sample edge. This can be attributed to the modulation of the screening current induced by the antidot rows as discussed in the Supplemental Material [31]. A similar mismatch was observed for all $H \leqslant H_{1}$, in which case the response is dominated by the Meissner effect rather than by
TABLE I. Elastic coupling constants (in $\mathrm{pN} / \mu \mathrm{m}$ ) due to antidots $\left(\kappa_{p}\right)$, random pinning $\left(\kappa_{r 0}\right)$, and vortex caging $\left(\kappa_{v}\right)$, and thermal hopping time $(\tau)$ calculated for both experimental temperatures using the fitting results of Fig. 7 .

\begin{tabular}{lcccc}
\hline \hline$T(\mathrm{~K})$ & $\kappa_{p}$ & $\kappa_{r 0}$ & $\kappa_{v}$ & $\omega \tau$ \\
\hline 6.7 & 20.6 & 0.269 & 0.00706 & 599 \\
6.9 & 4.44 & 0.0967 & 0.00424 & 0.0739 \\
\hline \hline
\end{tabular}

vortex motion. For higher fields, strong fluctuations induced by the interstitial vortices shaking within the scanning area can be observed in the experimental data, which of course is not captured by our mean field model. In spite of this, the fact that the use of only two fitting parameters makes it possible to capture the main trends of our experimental results is a compelling evidence that the model describes the main physics of the vortex dynamics in our sample.

In order to better understand the different dynamical behaviors observed in the different temperatures used in the experiments, we give in Table I the values of the coupling constants calculated for both temperatures using the fitting parameters. Notice that in both cases $\kappa_{p} \gg \kappa_{r 0} \gg \kappa_{v}$. However, thanks to the exponential dependence of the hopping time on the pinning parameters [cf. Eq. (13)], the product $\omega \tau$ is four orders of magnitude larger at $6.7 \mathrm{~K}(\omega \tau=599)$ than at $6.9 \mathrm{~K}(\omega \tau=0.0739)$. Therefore, for $6.9 \mathrm{~K}$ the vortices can hop several (about 13) times within one cycle of the excitation force, resulting in a strongly dissipative, creeplike motion and, consequently, in a prominent out-of-phase component of the ac screening length and the sample permeability. On the other hand, for $6.7 \mathrm{~K}$, they keep trapped essentially at the same pinning site and their motion is nearly reversible, leading to a virtually real ac screening length and, thereby, to an essentially inductive sample response.

If the temperature is further increased, one may expect that vortices hop so many times within each excitation cycle that, effectively, the random landscape is washed out. Only then, the caging of interstitial vortices dominates the response. To better illustrate this point, we consider, for simplicity, the case of $H_{d c}=2 H_{1}$ and take the limit of strong artificial pinning, that is $\alpha_{p} \gg \alpha_{r}, \alpha_{v}$, for which vortices in the artificial traps are essentially immobile and the low-frequency response function takes the simpler form

$$
\mu_{v}(\omega) \simeq \frac{\phi_{0}}{2}\left(\alpha_{v}+\frac{\alpha_{r 0}}{1-i / \omega \tau}\right)^{-1},
$$

where the factor $1 / 2$ reflects the fact that only half the vortex lattice (interstitial vortices) is moving, while the other half is immobile. This equation makes it clear that when $\omega \tau \rightarrow \infty$ (low temperature limit), the response is purely inductive and characterized by an effective spring constant $\alpha_{L}=\alpha_{v}+\alpha_{r 0}$. Since typically $\alpha_{r 0} \ll \alpha_{v}$, the response will be dominated by material defects such as in the conventional Campbell regime. In the other limit, $\omega \tau \rightarrow \infty$ (high temperatures), $\mu_{v}(\omega)$ is again purely inductive, but now $\alpha_{L}=\alpha_{v}$ and the response is dominated by the cage potential. Therefore, by controlling either the temperature or the excitation frequency one can fine-tune the mean number of thermal vortex hops 


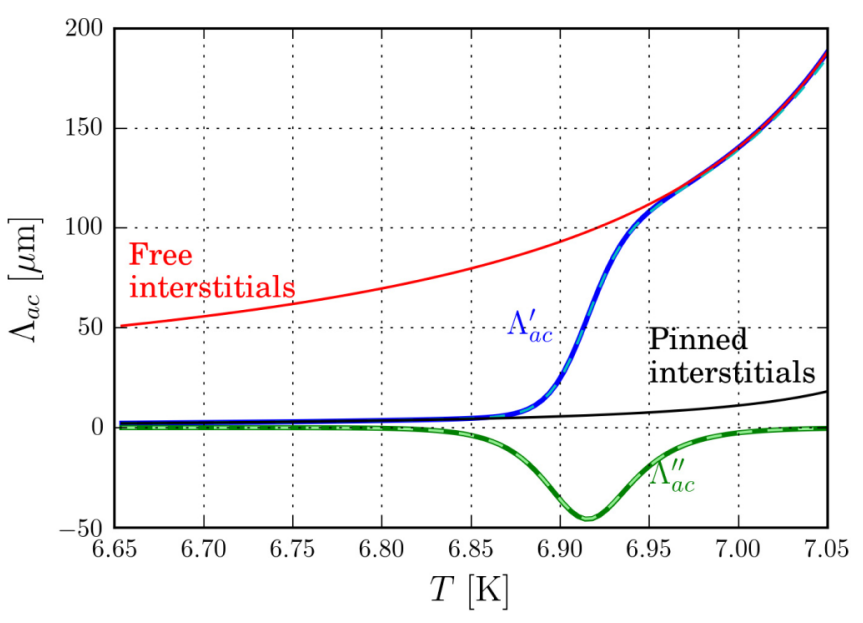

FIG. 9. Temperature dependence of the real (thick blue line) and imaginary (thick green line) components of the effective ac penetration depth calculated for the sample under excitation frequency $77.123 \mathrm{~Hz}$ using Eq. (16). Light dashed lines correspond to the approximate Eq. (21). For comparison, we also plot $\Lambda_{a c}(T)$ for the case where random pinning is absent (thin red line) and the case where thermal fluctuations are ignored (thin black line).

and, thereby, switch between two very different dynamical regimes: a low mobility one, dominated by pinning due to sample inhomogeneities, and a highly mobile one, dominated by a pinning mechanism based on the cage effect.

Figure 9 shows the ac screening length of the sample, $\Lambda_{a c}$, for an excitation frequency $77.123 \mathrm{~Hz}$, calculated for a wide temperature window using Eqs. (5) and (16), the formulas for the coupling constants [Eqs. (17)-(20)], and the values found for the fitting parameters, $c_{v}=0.09$ and $c_{r}=0.019$. The solid thick lines were obtained using the full equation (16), with $n=2$, while dashes represent the large $\alpha_{p}$ approximation [Eq. (21)]. The transition between the high-temperature, highly mobile regime, with a large ac penetration depth, to the lowtemperature frozen state, where $\Lambda_{a c} \approx \Lambda$, is very clear. This transition is accompanied by a peak in the imaginary part of $\Lambda_{a c}$, resulting in the observed dissipative component of the sample response at $T=6.9 \mathrm{~K}$.

It is worth mentioning that this effect is not restricted to nanostructured superconductors. In fact, Eq. (21) can well represent the low frequency response of a vortex subject to any confining background potential. For instance, a similar resonant dissipation has been observed experimentally for the dynamical response of a single vortex in a plain $\mathrm{Pb}$ microstrip [9]. In this system, dc Meissner currents and the interaction with a nearby vortex produced a background confining potential which adds to the random pinning landscape. This result cannot be explained in terms of a single Labusch constant. Rather, an additional spring constant representing the background confining potential is a crucial ingredient for cutting off the divergence of the complex response function at high temperatures (when pinning is washed out), thus resulting in the observed dissipation peak.

\section{CONCLUSION}

In summary, we have conducted scanning susceptibility microscopy (SSM) experiments that revealed the interplay between the different energy scales associated to the linear response of vortices in a nanostructured superconducting strip. For that, we took profit of the used local character of the SSM technique to systematically separate the contribution of vortices and screening currents to the sample response. The behavior of the inductive and dissipative components of the vortex response near the sample edge was found to be consistent with a Meissner-like state in which the external ac field is screened within a well-defined complex screening length, $\Lambda_{a c}$. By introducing a simple model which takes into account all relevant elastic couplings in this system (namely, vortex-antidot, vortex-vortex, and vortex-random pinning couplings), as well as thermal activation through weak pinning sites, we derived an expression for $\Lambda_{a c}$ in terms of the respective mean-field elastic constants. This allowed us to successfully fit the experimental data for different temperatures and fields and, thereby, to quantify the relevance of each elastic constant to the system response in the different experimental conditions.

We have found that this system, excited at the low frequency of $77.123 \mathrm{~Hz}$, is particularly sensible to thermal fluctuations at temperatures close to $T_{c}=7.2 \mathrm{~K}$. At $6.7 \mathrm{~K}$ the interstitial vortices are essentially frozen up by quenched disorder, whereas at the slightly larger temperature of $6.9 \mathrm{~K}$ they become highly mobile, as evidenced by the strong enhancement of the ac screening length. We attribute this drastic change of behavior at nearby temperatures to the exponential character of thermal activation through quenched disorder, which for $6.9 \mathrm{~K}$ allows interstitial vortices to thermally hop several times within each excitation cycle, thus rendering the observed high mobility. At $6.7 \mathrm{~K}$, the hopping time is just much larger than the excitation period, so that at this temperature the interstitial vortices become effectively pinned by material defects. This result highlights the importance of thermal relaxation in low- $T_{c}$ nanostructured superconductors and thereby of the time scales of experiments performed on these materials.

\section{ACKNOWLEDGMENTS}

This work was partially supported by the Brazilian funding agencies CNPq and FACEPE, under the Grants No. APQ1381-1.05/12 and No. APQ-0598/1.05-08, the Methusalem Funding by the Flemish government, the Fund for Scientific Research-Flanders (FWO-Vlaanderen), and the Fonds de la Recherche Scientifique F.R.S.-FNRS. The work of A.V.S. is partially supported by "Mandat d'Impulsion Scientifique" MIS F.4527.13 of the F.R.S.-FNRS. J.B. acknowledges support from F.R.S.-FNRS (Research Fellowship).
[1] G. Blatter, M. V. Feigel'man, V. B. Geshkenbein, A. I. Larkin, and V. M. Vinokur, Vortices in high-temperature superconductors, Rev. Mod. Phys. 66, 1125 (1994).
[2] C. J. van der Beek, V. B. Geshkenbein, and V. M. Vinokur, Linear and nonlinear ac response in the superconducting mixed state, Phys. Rev. B 48, 3393 (1993). 
[3] B. Raes, C. C. de Souza Silva, A. V. Silhanek, L. R. E. Cabral, V. V. Moshchalkov, and J. Van de Vondel, Closer look at the low-frequency dynamics of vortex matter using scanning susceptibility microscopy, Phys. Rev. B 90, 134508 (2014).

[4] M. Nikolo, Superconductivity: A guide to alternating current susceptibility measurements and alternating current susceptometer design, Am. J. Phys. 63, 57 (1995).

[5] F. Gömöry, Characterization of high-temperature superconductors by ac susceptibility measurements, Supercond. Sci. Technol. 10, 523 (1997).

[6] M. Marchevsky, M. J. Higgins, and S. Bhattacharya, Driven Dynamics of the Vortex-Phase Mixture Near the Peak Effect: The Vortex Capacitor, Phys. Rev. Lett. 88, 087002 (2002).

[7] M. P. DeFeo and M. Marchevsky, Localized ac response and stochastic amplification in a labyrinthine magnetic domain structure in a yttrium iron garnet film, Phys. Rev. B 73, 184409 (2006).

[8] R. B. G. Kramer, G. W. Ataklti, V. V. Moshchalkov, and A. V. Silhanek, Direct visualization of the Campbell regime in superconducting stripes, Phys. Rev. B 81, 144508 (2010).

[9] B. Raes, J. Van de Vondel, A. V. Silhanek, C. C. de Souza Silva, J. Gutierrez, R. B. G. Kramer, and V. V. Moshchalkov, Local mapping of dissipative vortex motion, Phys. Rev. B 86, 064522 (2012).

[10] A. T. Fiory, A. F. Hebard, and S. Somekh, Critical currents associated with the interaction of commensurate flux-line sublattices in a perforated Al film, Appl. Phys. Lett. 32, 73 (1978).

[11] M. G. Blamire, Flux vortex dynamics and electric fields in matched pinning systems, J. Low Temp. Phys. 68, 335 (1987).

[12] M. Baert, V. V. Metlushko, R. Jonckheere, V. V. Moshchalkov, and Y. Bruynseraede, Composite Flux-line Lattices Stabilized in Superconducting Films by a Regular Array of Artificial Defects, Phys. Rev. Lett. 74, 3269 (1995).

[13] V. Metlushko, U. Welp, G. W. Crabtree, Zhao Zhang, S. R. J. Brueck, B. Watkins, L. E. DeLong, B. Ilic, K. Chung, and P. J. Hesketh, Nonlinear flux-line dynamics in vanadium films with square lattices of submicron holes, Phys. Rev. B 59, 603 (1999).

[14] V. Metlushko, U. Welp, G. W. Crabtree, R. Osgood, S. D. Bader, L. E. DeLong, Zhao Zhang, S. R. J. Brueck, B. Ilic, K. Chung, and P. J. Hesketh, Interstitial flux phases in a superconducting niobium film with a square lattice of artificial pinning centers, Phys. Rev. B 60, R12585 (1999).

[15] A. V. Silhanek, S. Raedts, M. Lange, and V. V. Moshchalkov, Field-dependent vortex pinning strength in a periodic array of antidots, Phys. Rev. B 67, 064502 (2003).

[16] S. Raedts, A. V. Silhanek, M. J. Van Bael, and V. V. Moshchalkov, Flux-pinning properties of superconducting films with arrays of blind holes, Phys. Rev. B 70, 024509 (2004).

[17] A. V. Silhanek, S. Raedts, M. J. Van Bael, and V. V. Moshchalkov, Dynamic regimes in films with a periodic array of antidots, Eur. Phys. J. B 37, 19 (2004).

[18] A. V. Silhanek, S. Raedts, M. J. Van Bael, and V. V. Moshchalkov, Experimental determination of the number of flux lines trapped by microholes in superconducting samples, Phys. Rev. B 70, 054515 (2004).

[19] A. V. Silhanek, S. Raedts, and V. V. Moshchalkov, Paramagnetic reentrance of ac screening: Evidence of vortex avalanches in $\mathrm{Pb}$ thin films, Phys. Rev. B 70, 144504 (2004).
[20] S. Raedts, A. V. Silhanek, V. V. Moshchalkov, J. Moonens, and L. H. A. Leunissen, Crossover from intravalley to intervalley vortex motion in type-II superconductors with a periodic pinning array, Phys. Rev. B 73, 174514 (2006).

[21] E. T. Filby, A. A. Zhukov, P. A. J. de Groot, M. A. Ghanem, P. N. Bartlett, and V. V. Metlushko, Shape induced anomalies in vortex pinning and dynamics of superconducting antidot arrays with spherical cavities, Appl. Phys. Lett. 89, 092503 (2006).

[22] M. Motta, F. Colauto, R. Zadorosny, T. H. Johansen, R. B. Dinner, M. G. Blamire, G. W. Ataklti, V. V. Moshchalkov, A. V. Silhanek, and W. A. Ortiz, Visualizing the ac magnetic susceptibility of superconducting films via magneto-optical imaging, Phys. Rev. B 84, 214529 (2011).

[23] C. Chiliotte, G. Pasquini, V. Bekeris, J. E. Villegas, C.-P. $\mathrm{Li}$, and I. K. Schuller, Vortex lattice dynamics in $\mathrm{Nb}$ films with competing intrinsic random and artificial periodic pinning, Supercond. Sci. Technol. 24, 065008 (2011).

[24] C. C. de Souza Silva, J. A. Aguiar, and V. V. Moshchalkov, Linear ac dynamics of vortices in a periodic pinning array, Phys. Rev. B 68, 134512 (2003).

[25] A. I. Larkin and Yu. N. Ovchinnikov, Influence of inhomogeneities on superconductor properties, Zh. Eksp. Teor. Fiz. 61, 1221 (1971) [Sov. Phys.-JETP 34, 651 (1972)].

[26] E. H. Brandt, Thin superconductors in a perpendicular magnetic ac field: General formulation and strip geometry, Phys. Rev. B 49, 9024 (1994).

[27] E. H. Brandt, Theory of type-II superconductors with finite london penetration depth, Phys. Rev. B 64, 024505 (2001).

[28] M. Tinkham, Introduction to Superconductivity, 2nd ed. (McGraw-Hill, New York, 1996).

[29] M. W. Coffey and J. R. Clem, Unified Theory of Effects of Vortex Pinning and Flux Creep Upon the rf Surface Impedance of Type-II Superconductors, Phys. Rev. Lett. 67, 386 (1991).

[30] E. H. Brandt, Penetration of Magnetic ac Fields into Type-II Superconductors, Phys. Rev. Lett. 67, 2219 (1991).

[31] See Supplemental Material at http://link.aps.org/supplemental/ 10.1103/PhysRevB.94.024516 for a discussion concerning the effects of a non-uniform screening length and additional details on the experimental determination of the penetration depth and probe height.

[32] D. Yu. Vodolazov and I. L. Maksimov, Distribution of the magnetic field and current density in superconducting films of finite thickness, Physica C: Supercond. 349, 125 (2001).

[33] B. L. T. Plourde, D. J. Van Harlingen, D. Yu. Vodolazov, R. Besseling, M. B. S. Hesselberth, and P. H. Kes, Influence of edge barriers on vortex dynamics in thin weak-pinning superconducting strips, Phys. Rev. B 64, 014503 (2001).

[34] L. Embon, Y. Anahory, A. Suhov, D. Halbertal, J. Cuppens, A. Yakovenko, A. Uri, Y. Myasoedov, M. L. Rappaport, M. E. Huber, A. Gurevich, and E. Zeldov, Probing dynamics and pinning of single vortices in superconductors at nanometer scales, Sci. Rep. 5, 7598 (2015).

[35] J. Brisbois, B. Raes, J. Van de Vondel, V. V. Moshchalkov, and A. V. Silhanek, Determination of the magnetic penetration depth in a superconducting Pb film, J. Appl. Phys. 115, 103906 (2014).

[36] There is a misprint in Eqs. (4) and (5) of Ref. [24]. The sign of the $\alpha_{v}$ term in both equations is supposed to be a minus. A similar correction is needed in Eq. (8). In the right hand side, 
the imaginary part is supposed to be negative. All plots were obtained from the correct version of these equations.

[37] A. M. Campbell, The response of pinned flux vortices to low-frequency fields, J. Phys. C: Solid State Phys. 2, 1492 (1969).

[38] J. Gutierrez, B. Raes, J. Van de Vondel, A. V. Silhanek, R. B. G. Kramer, G. W. Ataklti, and V. V. Moshchalkov, First vortex entry into a perpendicularly magnetized superconducting thin film, Phys. Rev. B 88, 184504 (2013).

[39] M. Marchevsky, P. H. Kes, and J. Aarts, Determination of the quenching temperature for the vortex lattice in field-cooling decoration experiments, Physica C: Supercond. Appl. 282-287, 2083 (1997).

[40] S. J. Bending, Local magnetic probes of superconductors, Adv. Phys. 48, 449 (1999).

[41] C. Reichhardt and N. Grønbech-Jensen, Critical currents and vortex states at fractional matching fields in superconductors with periodic pinning, Phys. Rev. B 63, 054510 (2001).

[42] S. B. Field, S. S. James, J. Barentine, V. Metlushko, G. Crabtree, H. Shtrikman, B. Ilic, and S. R. J. Brueck, Vortex Configurations, Matching, and Domain Structure in Large Arrays of Artificial Pinning Centers, Phys. Rev. Lett. 88, 067003 (2002).

[43] A. N. Grigorenko, S. J. Bending, M. J. Van Bael, M. Lange, V. V. Moshchalkov, H. Fangohr, and P. A. J. de Groot, Symmetry Locking and Commensurate Vortex Domain Formation in Periodic Pinning Arrays, Phys. Rev. Lett. 90, 237001 (2003).
[44] C. C. de Souza Silva, L. R. E. Cabral, and J. Albino Aguiar, Vortex dynamics in superconducting films: Comensurability and surface effects, Phys. Status Solidi A 187, 209 (2001).

[45] C. P. Bean and J. D. Livingston, Surface Barrier in Type-II Superconductors, Phys. Rev. Lett. 12, 14 (1964).

[46] J. R. Clem, A model for flux pinning in superconductors, in Low Temperature Physics-LT 13 (Springer, New York, 1974), pp. 102-106.

[47] C. C. de Souza Silva, L. R. E. Cabral, and J. A. Aguiar, Flux penetration, matching effect, and hysteresis in homogeneous superconducting films, Phys. Rev. B 63, 134526 (2001).

[48] M. V. Indenbom, H. Kronmüller, T. W. Li, P. H. Kes, and A. A. Menovsky, Equilibrium magnetic properties and Meissner expulsion of magnetic flux in $\mathrm{Bi}_{2} \mathrm{Sr}_{2} \mathrm{CaCu}_{2} \mathrm{O}_{\mathrm{x}}$ single crystals, Physica C 222, 203 (1994).

[49] M. Benkraouda and J. R. Clem, Magnetic hysteresis from the geometrical barrier in type-II superconducting strips, Phys. Rev. B 53, 5716 (1996).

[50] In Ref. [35], the Meissner state was studied for fields just below and at the penetration field, for which cases the screening current at the edge approaches the depairing current. This induces strong depreciation of the order parameter near the sample edge, which, in turn, lead to an enhancement of the penetration depth by a factor of 1.84 at this region [51].

[51] K. Fossheim and A. Sudbø, Superconductivity: Physics and Applications (John Wiley \& Sons, New York, 2005), p. 234. 